\title{
Surface-enhanced Raman Scattering Enhancement Factors for RNA Mononucleotides on Silver Nanoparticles
}

\author{
Snežana Miljanić, ${ }^{1, *}$ Marina Ratkaj, ${ }^{2}$ Igor Avdejev, ${ }^{2}$ Karlo Meglić, ${ }^{1}$ Adriana Kenđel ${ }^{1}$
}

\author{
${ }_{1}$ Division of Analytical Chemistry, Department of Chemistry, Faculty of Science, University of Zagreb, Horvatovac 102a, HR-10000 Zagreb, Croatia \\ 2 PLIVA Croatia, Teva Pharmaceutical Industries Ltd., Research and Development, Prilaz baruna Filipovića 29, HR-10000 Zagreb, Croatia \\ * Corresponding author's e-mail address: miljanic@chem.pmf.hr \\ RECEIVED: March 15, 2015 * REVISED: July 15, 2015 * ACCEPTED: September 15, 2015
}

THIS PAPER IS DEDICATED TO DR. SVETOZAR MUSiĆ ON THE OCCASION OF HIS 70 ${ }^{\mathrm{TH}}$ BIRTHDAY

\begin{abstract}
Surface-enhanced Raman scattering (SERS) enhancement factors (EF) were evaluated for RNA mononucleotides: adenosine 5'-monophosphate (AMP), guanosine 5'-monophosphate (GMP), cytidine 5'-monophosphate (CMP) and uridine 5'-monophosphate (UMP), on silver nanoparticles, which differed in shape (nanospheres, nanostars) and stabilizing anionic layer (chlorides, citrates) on the metal surface. In freshly prepared silver colloids the enhanced Raman scattering was observed for all the RNA mononucleotides on the chloride coated silver nanospheres, Ag_Cl nsp $\left(E F \approx 10^{4}\right)$, for AMP only on the citrate coated silver nanospheres, Ag_cit nsp $\left(E F \approx 10^{3}\right)$, while not obtained at all for any of the mononucleotides on the citrate stabilized silver nanostars, $\mathrm{Ag}_{\mathbf{1}}$ cit nst. Upon aggregation, the SERS activity of all the silver colloids increased, whereby the purine mononucleotides, AMP and GMP, more strongly scattered radiation on Ag_Cl nsp, and the pyrimidine mononucleotides, CMP and UMP, on Ag_cit nsp. Regardless of the silver nanoparticles, the higher $E F s$ were evaluated for AMP and GMP $\left(E F\right.$ up to $\left.5 \times 10^{6}\right)$, than for $\mathrm{CMP}$ and $\mathrm{UMP}\left(E F \approx 5 \times 10^{4}\right)$.
\end{abstract}

Keywords: surface-enhanced Raman scattering, enhancement factor, RNA mononucleotide, silver nanoparticles, shape, anions.

\section{INTRODUCTION}

$\mathbf{S}$ URFACE-ENHANCED Raman scattering (SERS) spectroscopy is a powerful vibrational spectroscopy technique that allows for highly sensitive structural detection of low concentration molecules in vicinity to nanostructured metal surfaces. ${ }^{[1]}$ Owing to recent developments in design and fabrication of plasmonic substrates, essential for the Raman scattering enhancement, application of the SERS spectroscopy in analytical, biophysical and life sciences constantly increases. ${ }^{[2]}$ The enhancement of the Raman scattering is associated with two mechanisms: an electromagnetic mechanism and a chemical mechanism. The electromagnetic enhancement mechanism results from the amplification of the radiation by excitation of the localized surface plasmon resonance (LSPR), while the chemical enhancement mechanism involves charge transfer when the excitation energy is resonant with the metal-molecule charge transfer electronic states. Although electromagnetic mechanism dominantly contributes to the overall enhancement, the total SERS enhancement factor with respect to the normal Raman signal, in most cases is the product of both mechanisms, reaching up to $10^{10}$.

The prerequisite for the Raman scattering enhancement is adsorption of the molecules onto or near a roughened metal surface. In order to enable effective surface adsorption, various SERS active substrates have been developed, ranging from electrodes, metal island films, to commonly used metal colloids. ${ }^{[3]}$ Colloidal suspensions seem to be particularly attractive due to rather simple and inexpensive preparation procedures, average SERS enhancement up to $10^{6}$ and fair reproducibility. ${ }^{[4]}$ In addition, the maximal surface enhancement of the scattered radiation can be achieved by adjusting the colloidal nanoparticles properties. The majority of the metal nanoparticles have been made of gold $(\mathrm{Au})$ and silver $(\mathrm{Ag})$, both metals being characterized 


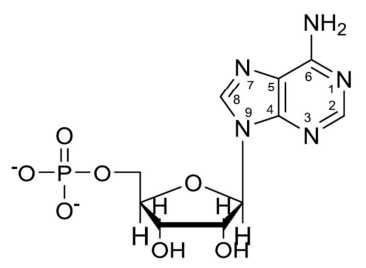

AMP

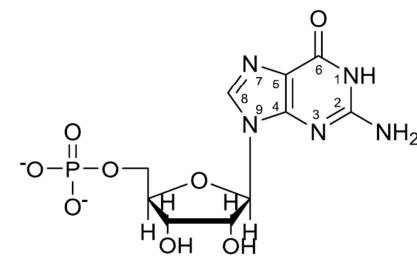

GMP<smiles>Nc1ccn(C(O)OC(O)COP(=O)(O)O)c(=O)n1</smiles>

CMP<smiles>O=c1ccn(C2OC(COP(=O)([O-])[O-])[C@@H](O)[C@H](O)[C@H]2O)c(=O)[nH]1</smiles>

UMP

Scheme 1. Molecular structures of the RNA mononucleotides.

by the surface plasmon resonance in the Vis region. However, experimental and theoretical studies on plasmonic substrates implied that, beside the metal type, morphology of the nanoparticles strongly affects LSPR, resulting in enhancement of the Raman scattering upon Vis as well as NIR excitation. ${ }^{[5]}$ Given that subtle changes in the nanostructure alter the local field enhancement and lead to significant LSPR shifts, much emphasis has been put on controlling the shape of the metallic nanoparticles. Hence a huge intensification of the electromagnetic field has been observed for the anisotropic metal nanoparticles. ${ }^{[5]}$ Among non-spherically shaped nanoparticles nanostars have been particularly attractive due to the tip-associated plasmon resonance, which can be synthetically tuned into the NIR region. ${ }^{[6]}$ In addition to the shape effect, the plasmon frequency for the single nanoparticle decreases as the particle size increases as well as the nanoparticles aggregate. ${ }^{[4,7]}$ Except for the change in the surface plasmon resonance, nanoparticles aggregation leads to generation of enormous electric field in interparticle gaps. To control nanoparticle aggregation, an aggregating agent is usually introduced into the colloidal suspension, where either by reacting with the metal surface or by coating the nanoparticle, reduces the surface charge. Except for stabilization of the metallic nanoparticles in the suspension, the surface coatings is of the utmost importance for the adsorption of the molecules on the colloidal nanoparticles. ${ }^{[8]}$ If colloidal nanoparticles and molecules had charges of the same sign, adsorption process could be strongly hindered, or even completely prevented.

Here, we report on an influence of the nanoparticle properties on the surface-enhanced Raman scattering of four RNA mononucleotides: adenosine 5'-monophosphate (AMP), guanosine 5'-monophosphate (GMP), cytidine 5'monophosphate (CMP) and uridine 5'-monophosphate (UMP) (Scheme 1). Given that majority of the effective metal nanoparticles carry a negative charge due to the layer of stabilizing anions on their surface, hence facilitating adsorption of the positively charged molecules, this work was aimed at the study of the Raman scattering of the anionic species from the enhancing surface of the same charge. With regard to the strongest enhancement generally observed for the silver nanoparticles, species on the metal surface, responsible for the negative surface charge, were varied on the $\mathrm{Ag}$ nanospheres, whereby those with the surface layer of the citrate $\mathrm{e}^{[9,10]}$ and chloride ${ }^{[11]}$ ions were employed. Furthermore, the influence of the nanoparticle shape was studied using silver nanospheres ${ }^{[10]}$ and nanostars, ${ }^{[6]}$ both being stabilized by citrates on the metal surface. Based on recent studies on label free detection of DNA using sulfate salts to aggregate silver nanoparticles, ${ }^{[12,13]}$ the effect of aggregation on SERS enhancement of the RNA mononucleotides was studied with sodium sulfate as an aggregating agent. Finally, a suitability of various $\mathrm{Ag}$ nanoparticles for the study of small, negatively charged biomolecules was evaluated by calculation of the analytical enhancement factor. ${ }^{[14]}$

\section{EXPERIMENTAL}

\section{Chemicals and Solutions}

Silver nitrate (Kemika), sodium hydroxide (Kemika), trisodium citrate (Kemika), hydroxylamine hydrochloride (Kemika) and hydroxylamine solution (50 $\mathrm{w} / \mathrm{w}$ in water) (Aldrich) were of analytical reagent grade and used as supplied for the silver colloids preparation. Sodium sulfate, used for aggregation of colloidal nanoparticles, was purchased from Kemika. Water was purified by passage through Milli-Q (Millipore) deionizing and filtration columns.

RNA mononucleotides: adenosine 5'-monophosphate disodium salt, guanosine $5^{\prime}$-monophosphate disodium salt, cytidine $5^{\prime}$-monophosphate disodium salt and uridine $5^{\prime}$-monophosphate disodium salt were purchased from Sigma-Aldrich. Stock solutions of the mononucleotides were prepared by dissolution of the substance in water of Milli-Q purity resulting in the final concentration of $1 \times 10^{-1} \mathrm{~mol} / \mathrm{L}$. These solutions were further on diluted to solutions of $2.5 \times 10^{-4}, 2.5 \times 10^{-5}, 2.5 \times 10^{-6}$ and $2.5 \times 10^{-7} \mathrm{~mol} / \mathrm{L}$, used for preparation of the samples for SERS measurements.

The aqueous stock solutions of the RNA mononucleotides $\left(1 \times 10^{-1} \mathrm{~mol} / \mathrm{L}\right)$ were used for the measurement of the Raman spectra. The working samples for the SERS measurements were prepared by mixing the silver colloid $(400 \mu \mathrm{L})$ 
with the RNA mononucleotide solution $(400 \mu \mathrm{L})$, followed by addition of either water $(200 \mu \mathrm{L})$ or the aggregating agent solution $(200 \mu \mathrm{L})$, which was $\mathrm{Na}_{2} \mathrm{SO}_{4}\left(1 \times 10^{-1} \mathrm{~mol} / \mathrm{L}\right)$. Final concentrations of the RNA mononucleotides in the measured samples were $1 \times 10^{-4}, 1 \times 10^{-5}, 1 \times 10^{-6}$ and $1 \times$ $10^{-7} \mathrm{~mol} / \mathrm{L}$.

To obtain a measurable absorption of prepared silver colloidal suspensions, freshly prepared colloids $(1 \mathrm{~mL})$ were diluted with Milli-Q water $(2 \mathrm{~mL})$.

\section{Colloid Preparation}

Silver nanospheres stabilized by the surface chloride ions (Ag_Cl nsp) were prepared according to the reduction method of silver nitrate with hydroxylamine hydrochloride. ${ }^{[11]}$ Hydroxylamine hydrochloride $(0.017 \mathrm{~g})$ was dissolved in Milli-Q water $(10 \mathrm{~mL})$ with addition of sodium hydroxide ( $2 \mathrm{~mol} / \mathrm{L}, 0.15 \mathrm{~mL}$ ). A silver nitrate solution was prepared by dissolving silver nitrate $(0.017 \mathrm{~g})$ in Milli-Q water $(90 \mathrm{~mL})$. Alkaline hydroxylamine hydrochloride solution was added rapidly to the silver nitrate solution under vigorous stirring and kept stirring for $10 \mathrm{~min}$. For the resulting grey colloidal suspension a maximum at $414 \mathrm{~nm}$ was obtained in the UV/Vis absorption spectrum. A pH value of the prepared colloid was 6.7.

Silver nanospheres stabilized by the surface citrate ions (Ag_cit nsp) were prepared according to the modified Lee and Meisel reduction method with trisodium citrate. ${ }^{10}$ Aqueous solution of $1 \%(w / v)$ trisodium citrate $(2 \mathrm{~mL})$ was added to the boiling solution of $1 \times 10^{-3} \mathrm{~mol} / \mathrm{L}$ silver nitrate $(100 \mathrm{~mL})$ and kept boiling for 90 minutes. The resulting colloidal suspension was grey coloured, characterized by an absorption maximum at $426 \mathrm{~nm}$ and a $\mathrm{pH}$ value of 6.8 .

Silver nanostars (Ag_cit nst) were produced by the reduction of silver nitrate using two reducing agents: hydroxylamine in the first stage and citrate in the second stage of the preparation process. ${ }^{[6]}$ In the mixture of hydroxylamine solution $(0.06 \mathrm{~mol} / \mathrm{L}, 5 \mathrm{~mL})$ and $\mathrm{NaOH}$ $(0.05 \mathrm{~mol} / \mathrm{L}, 5 \mathrm{~mL})$, solution of silver nitrate $\left(1 \times 10^{-3} \mathrm{~mol} / \mathrm{L}\right.$, $90 \mathrm{~mL}$ ) was added dropwise under agitation. After $5 \mathrm{~min}$ of mixing, trisodium citrate $(1 \%(w / v), 1 \mathrm{~mL})$ was added and the mixture was stirred for the following $15 \mathrm{~min}$. For the obtained light grey suspension an absorption maximum at $389 \mathrm{~nm}$ was observed and a $\mathrm{pH}$ of 7.9 was measured.

\section{Instrumentation}

The Raman and SERS spectra were measured on a RamanRXN1 spectrometer (Kaiser Optical Systems), equipped with a laser diode emitting at $785 \mathrm{~nm}$ and a thermoelectrically cooled CCD detector. The samples were analyzed with a 100 $\mu \mathrm{m}$-thick probe of collection fibers. The spectra were acquired using a laser power of $400 \mathrm{~mW}$ in the $3450-150 \mathrm{~cm}^{-1}$ spectral range at a resolution of $4 \mathrm{~cm}^{-1}$ and were averaged over three scans, having an exposure time of $10 \mathrm{~s}$.
UV/Vis/NIR absorption measurements were carried out with an Analytik Jena spectrometer (model SPECORD 200). Absorption spectra were measured in the 200-1100 $\mathrm{nm}$ spectral range. Conventional quartz cells $(10 \mathrm{~mm} \times 10$ $\mathrm{mm}$ ) were used throughout.

Transmission electron micrographs (TEM) were taken on a Zeiss EM10 transmission electron microscope from a drop of a colloid on a carbon coated grid (copper, 100 mesh).

For the $\mathrm{pH}$ measurement, a Mettler Toledo $\mathrm{pH}$ meter (model FE20 FiveEasy) with a Mettler Toledo LE409 glass electrode was used. The $\mathrm{pH}$ meter was calibrated with standard aqueous buffer solutions of $\mathrm{pH}=7.00$ and 4.01 .

\section{RESULTS AND DISCUSSION}

\section{Silver Colloidal Suspensions}

To study the enhancement efficiency of the nanoparticles varying in the stabilizing ion layer on the silver surface, which affect the adsorption of the molecules on the enhancing metal surface, silver nanospheres covered by chloride (Ag_Cl nsp) and citrate (Ag_cit nsp) ions were prepared. These two types of anions differed in their structural bulkiness and binding affinity with the silver surface, chlorides being smaller and having a higher affinity for Ag than citrates. ${ }^{[15]}$ Both anions were introduced into the colloidal suspensions with the reducing agents during the colloid preparation process. They were adsorbed on the metal surface, providing enough charge to the nanoparticles to maintain them dispersed in the aqueous medium due to the inter-particle repulsion forces. Given that citrates also participated in the reduction reaction with the silver ions, it was very likely that beside citrates, their oxidation products, such as acetoacetic acid, also existed on the silver surface. ${ }^{[10]}$ Unlike the citrate ions, chlorides were not involved in the chemical reduction of silver, but only as counter ions formed the surface layer, stabilizing the produced metal nanoparticles in the suspension. ${ }^{[11]}$

In order to keep the same nanoparticle coating properties, and to study the influence of the nanoparticle morphology, citrate stabilized star-shaped nanoparticles (Ag_cit nst) were prepared. The citrate ions were added into the colloid preparation mixture to accelerate the reduction process in the second step of the nanostars synthesis, leading to the growth of long star arms. As in case of the silver nanospheres, citrates also participated in formation of the negatively charged layer on the metal surface, preventing the nanoparticles from precipitation.

To reveal the size and shape of the prepared silver nanoparticles, TEM images were taken (Figure 1). The spherical nanoparticles coated by both, chloride and citrate ions, had a diameter of 20-120 nm (Figures 1a and 1b). Unlike 

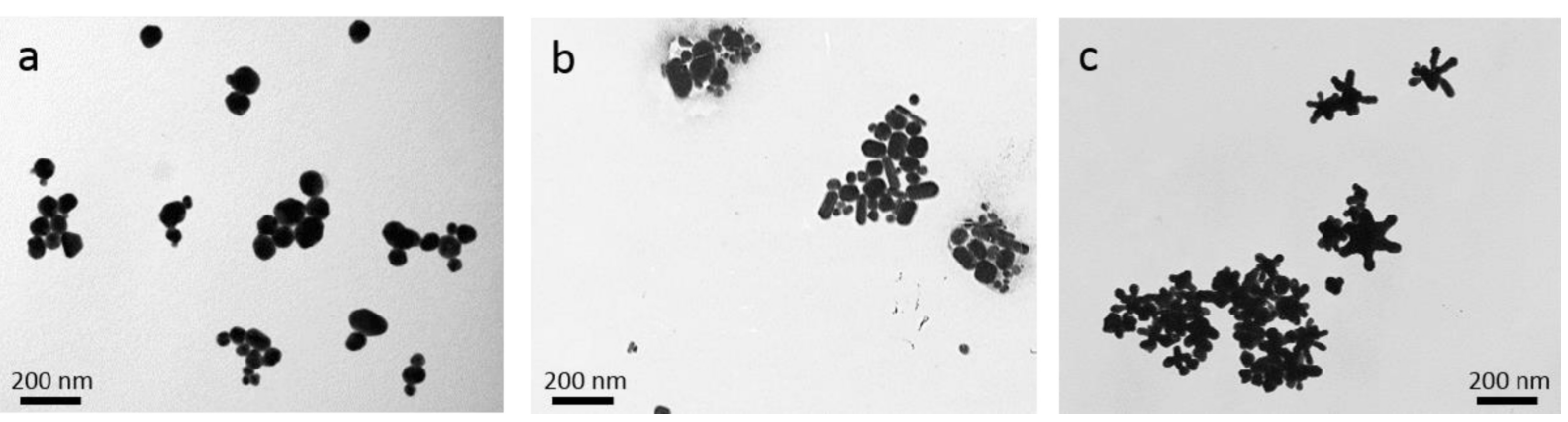

Figure 1. TEM micrographs of a) chloride coated silver nanospheres, Ag_Cl nsp, b) citrate coated silver nanospheres, $\mathrm{Ag} \_$cit nsp, and c) citrate coated silver nanostars, Ag_cit nst.

the uniformly shaped silver nanospheres with the chloride ions on their surface, more irregular forms and rods of 90-100 nm length were observed in the colloid prepared by the citrate reduction method (Figure $1 b$ ). The star-shaped nanoparticles consisted of the central core and several (5-9), 40-90 $\mathrm{nm}$ long arms, having an average diameter in the range of 100-220 nm (Figure 1c). Except for a few monodispersed nanoparticles, the majority of all the prepared silver nanoparticles formed aggregates.

In the UV/Vis/NIR extinction spectra of the colloidal suspensions a maximum around $400 \mathrm{~nm}$, typical of the silver plasmon resonance, was obtained (Figure 2). Regardless of the anionic coating, the spherically shaped nanoparticles, $\mathrm{Ag} \_\mathrm{Cl} n s p$ and $\mathrm{Ag}$ _cit nsp, gave rise to similar spectra with the maxima at 414 and $426 \mathrm{~nm}$, respectively. Unlike the spectra of the silver nanospheres, however, in the UV/Vis/NIR spectrum of the silver nanostars, Ag_cit nst, a weak maximum at $389 \mathrm{~nm}$ was observed, accompanied by a strong extinction at longer wavelengths. The large extinction background was attributed to the various morphologies of the existing nanoparticles, which resulted from different number, shape and size of the star arms. ${ }^{[6]}$

All the prepared silver colloidal suspensions were of neutral $\mathrm{pH}$ value, i.e. the $\mathrm{pH}$ of $6.7,6.8$ and 7.9 , was measured for Ag_Cl nsp, Ag_cit nsp and Ag_cit nst, respectively.

\section{SERS Enhancement}

The silver nanoparticles prepared by reduction methods with citrate and hydroxylamine hydrochloride have been widely used as the SERS active substrates and found very effective for the SERS measurement of various positively charged species. ${ }^{[3,4]}$ The detection of the species carrying a negative charge, however, has not been as successful due to the electrostatic repulsive interactions between the analyte and the stabilizing anionic layer, preventing an efficient adsorption of the analyte molecules onto the metallic surface. To overcome these drawbacks, an aggregating agent is usually added into the colloid, neutralizing the surface charge on the one hand, and forming the nanoparticles aggregates with numerous "hotspots" of extremely strong electric fields, on the other hand.

Aiming at the study of the surface enhancement of the negatively charged molecules, the RNA mononucleotides were chosen as the model molecules for several reasons. (i) All four mononucleotides had the same structural moiety responsible for the anionic character of the molecule, the phosphate group, but differed in the nucleic base involved in the adsorption of the biomolecule onto the metal surface. (ii) The adsorption mechanisms of the RNA mononucleotides onto the citrate coated silver nanoparticles had been thoroughly studied and discussed in our previous work.[16] (iii) Since the mononucleotides are the building blocks of the nucleic acids, knowing the Raman scattering enhancement of the structural elements attached to the various SERS substrate would be beneficial in the future SERS measurements of biomacromolecules.

In order to compare enhancement efficiency of the studied silver nanoparticles for the SERS measurements of

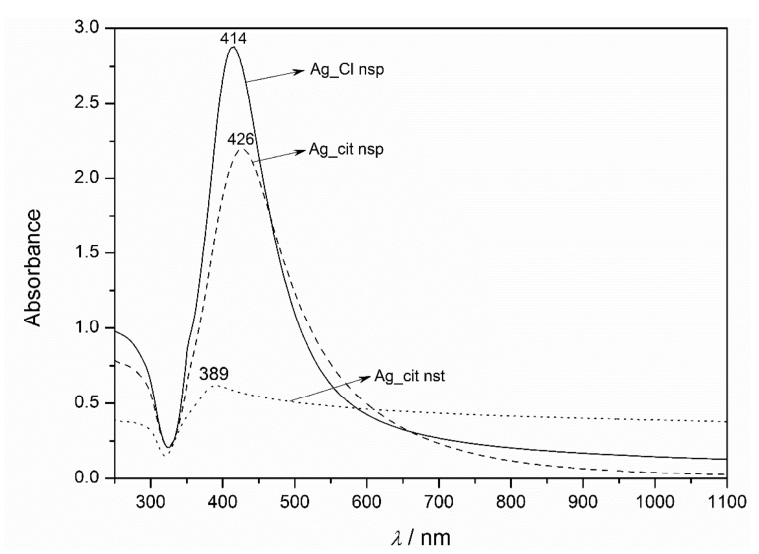

Figure 2. UV/Vis/NIR absorption spectra of the silver colloidal suspensions: Ag_Cl nsp, Ag_cit nsp and Ag_cit nst. 


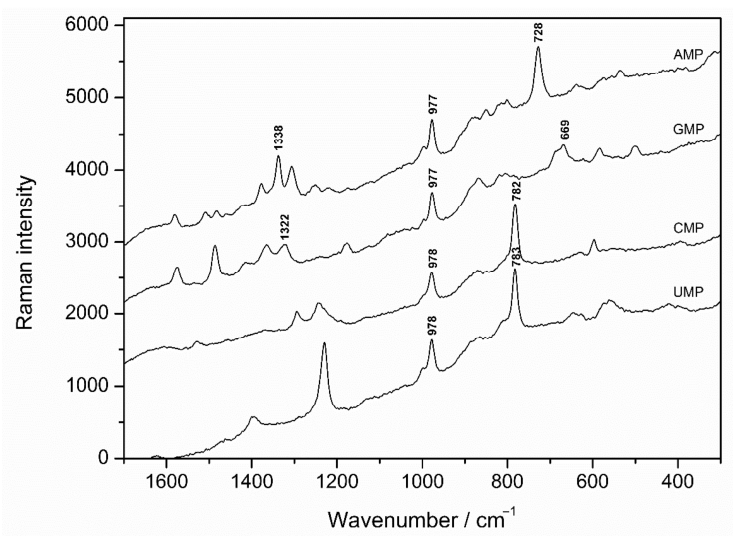

Figure 3. Raman spectra of the RNA mononucleotides $\left(1 \times 10^{-1} \mathrm{~mol} / \mathrm{L}\right)$ in aqueous solution. Excitation at $785 \mathrm{~nm}$. The spectra are displaced for visual clarity.

the RNA mononucleotides, an analytical enhancement factor, $E F$, was calculated. It is usually used to evaluate an enhancement of the Raman scattering obtained from an analyte adsorbed on a SERS active surface with respect to the normal Raman scattering of the same analyte in solution. ${ }^{[14]}$ The Raman and SERS measurements were conducted under the same experimental conditions, including laser wavelength, laser power, detector and spectrometer. The enhancement factor was calculated according to the following equation:

$$
E F=\frac{I_{\text {SERS }} / c_{\text {SERS }}}{I_{\text {RS }} / C_{\text {RS }}}
$$

where $l$ is the intensity of a band and $c$ is the analyte concentration in the solution (RS) or in the colloidal suspension (SERS), whereas subscripts RS and SERS refer to the Raman spectrum and SERS spectrum, respectively.

The Raman spectra of the RNA mononucleotides ( $1 \times$ $10^{-1} \mathrm{~mol} / \mathrm{L}$ ) in aqueous solution were acquired and the main vibrational bands tentatively assigned (Figure 3, Table 1). On first sight the obtained spectra reflected the complexity of the mononucleotides structure, being simpler in case of the pyrimidine mononucleotides, $\mathrm{CMP}$ and $\mathrm{UMP}$, and more complex in case of the purine mononucleotides, AMP and GMP. Nevertheless, in all the Raman spectra dominated bands assigned to the ring modes of the nucleic bases, except for a band at $977 \mathrm{~cm}^{-1}\left(978 \mathrm{~cm}^{-1}\right)$ common to the all mononucleotides, which was attributed to the symmetrical stretching of the phosphate group. ${ }^{[17]}$

The SERS spectra of the mononucleotides were measured in prepared silver colloids in the concentration range from $1 \times 10^{-7}$ to $1 \times 10^{-4} \mathrm{~mol} / \mathrm{L}$. Since the Raman scattering

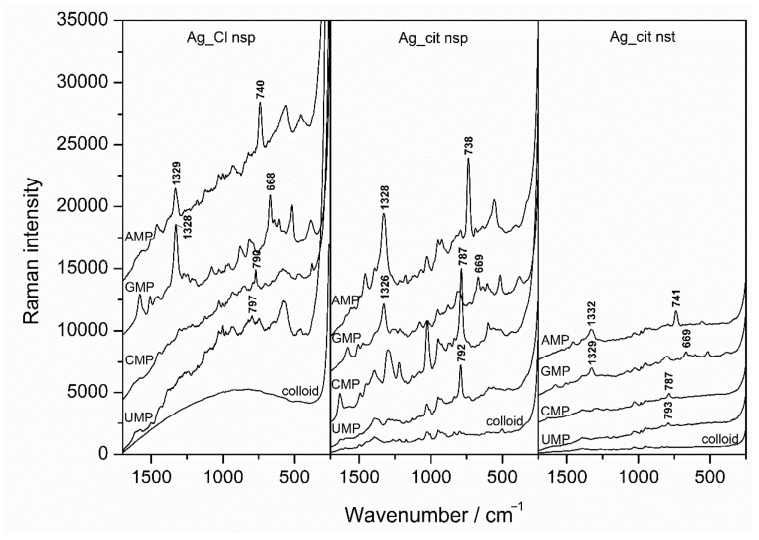

Figure 4. SERS spectra of the RNA mononucleotides $\left(1 \times 10^{-5}\right.$ $\mathrm{mol} / \mathrm{L}$ ) on the aggregated silver colloids: $\mathrm{Ag} \_\mathrm{Cl} n s p, \mathrm{Ag}_{\mathrm{C}} \mathrm{cit}$ nsp and Ag_cit nst, including the Raman spectra of the aggregated colloids. Excitation at $785 \mathrm{~nm}$. The SERS spectra are all shown with the same Raman intensity scale, but are displaced for visual clarity. The colloid Raman spectra are reduced by 10 times.

was poorly enhanced from the citrate stabilized silver nanospheres and not observed at all from the silver nanostars, sodium sulfate was added into the colloidal samples to induce the nanoparticles aggregation, followed by the SERS enhancement. The representative SERS spectra of the RNA mononucleotides $\left(1 \times 10^{-5} \mathrm{~mol} / \mathrm{L}\right)$ in the aggregated colloids are shown in Figure 4, and the assignment of the main vibrational bands is given in Table 1 . Based on a detailed analysis of the SERS spectra, adsorption mechanisms of the RNA mononucleotides on the silver nanoparticles had been thoroughly studied in our previous work and were discussed elsewhere. ${ }^{[16]}$

While selecting the appropriate band for the $E F$ calculation, a distinctive, intense and isolated band was preferred and as such observed in both, the Raman and SERS spectrum. Even though slightly overlapped by neighboring bands, particularly in the case of the guanine mononucleotide, the Raman bands at 728, 669, 782 and $783 \mathrm{~cm}^{-1}$, assigned to the aromatic ring breathing, were chosen for evaluating the Raman scattering enhancement of AMP, GMP, CMP and UMP, respectively. Given that involvement of the nucleic bases in the adsorption on the metal surface could affect the intensity of the studied bands, additional bands for the adenine $\left(1338 \mathrm{~cm}^{-1}\right)$ and guanine $\left(1322 \mathrm{~cm}^{-1}\right)$ containing mononucleotides were also analyzed for comparison.

In the freshly prepared silver colloids, not containing the aggregating agent, the enhanced Raman scattering was observed for all the RNA mononucleotides on the chloride coated silver nanospheres, for AMP only on the citrate 
Table 1. A tentative assignment of the main vibrational bands in the Raman spectra of the RNA mononucleotides in aqueous solution $\left(1 \times 10^{-1} \mathrm{~mol} / \mathrm{L}\right)$ and in the SERS spectra of RNA mononucleotides on the aggregated silver colloids $\left(1 \times 10^{-5} \mathrm{~mol} / \mathrm{L}\right)$

\begin{tabular}{|c|c|c|c|c|c|}
\hline \multirow{3}{*}{$\begin{array}{c}\text { RNA } \\
\text { mononucleotide }\end{array}$} & \multirow{3}{*}{ Raman } & \multicolumn{3}{|c|}{ Wavenumber $/ \mathrm{cm}^{-1}$} & \multirow{3}{*}{ Assignment } \\
\hline & & & SERS & & \\
\hline & & Ag_Clnsp & Ag_cit nsp & Ag_cit nst & \\
\hline \multirow{20}{*}{ AMP } & $1580 w$ & & & & $v$ ring $(6)$ \\
\hline & $1509 w$ & & & & $v$ ring $(6)$ \\
\hline & $1482 w$ & & & & $v$ ring $(6)$ \\
\hline & & $1458 w$ & $1457 w$ & $1454 w$ & $v$ ring $(6)$ \\
\hline & $1377 w$ & & $1390 w$ & $1384 w$ & $v$ ring $(\mathrm{C} 6-\mathrm{N} 1)$ \\
\hline & $1338 \mathrm{~m}$ & $1329 \mathrm{~m}$ & $1328 \mathrm{~s}$ & $1332 \mathrm{~s}$ & $v$ ring $(5)$ \\
\hline & $1306 \mathrm{~m}$ & & & & $v$ ring (5) \\
\hline & $1251 \mathrm{w}$ & & & & $v \mathrm{C} 6-\mathrm{NH}_{2}$ \\
\hline & $1218 v w$ & & & & $v$ ring (5) \\
\hline & & & $1027 w$ & $1029 w$ & citrates \\
\hline & $998 \mathrm{sh}$ & 1001 vw & & & $v_{\text {as }} \mathrm{C}-\mathrm{O}$ (ribose) \\
\hline & 977 m & & & & $\mathrm{v}_{\mathrm{s}} \mathrm{PO}_{3}{ }^{2-}$ \\
\hline & & & $951 w$ & $948 w$ & citrates \\
\hline & & 924 vw & $924 w$ & $921 w$ & $\delta$ ring $(6)$ \\
\hline & $851 w$ & $849 v w$ & & & $v_{s} \mathrm{C}-\mathrm{O}$ (ribose) \\
\hline & $728 \mathrm{~s}$ & $740 \mathrm{~s}$ & $738 s$ & $741 \mathrm{~s}$ & ring breathing \\
\hline & $638 v w$ & & & & $\delta$ ring (6) \\
\hline & $536 w$ & $564 w$ & $558 \mathrm{~m}$ & $557 w$ & $\delta$ ring $(6)$ \\
\hline & & 237 vs & & & $v \mathrm{Ag}-\mathrm{Cl}$ \\
\hline & & & 223 vs & 225 vs & $v \mathrm{Ag}-\mathrm{N}$ \\
\hline \multirow{18}{*}{ GMP } & $1574 \mathrm{~m}$ & $1579 w$ & $1579 w$ & $1578 w$ & $v$ ring $(6)$ \\
\hline & $1486 \mathrm{~s}$ & $1508 w$ & $1507 w$ & 1506 w & $v$ ring (6); $v$ ring (5) \\
\hline & & $1453 w$ & 1472 w & & $v$ ring (5) \\
\hline & $1414 w$ & & & & $v$ ring (6) \\
\hline & $1365 \mathrm{~m}$ & & & & $\delta_{\text {ip }} \mathrm{N} 1-\mathrm{H} ; v$ ring $(6)$ \\
\hline & $1322 \mathrm{~m}$ & $1328 \mathrm{~s}$ & $1326 \mathrm{~s}$ & $1329 \mathrm{~s}$ & $v$ ring (5) \\
\hline & 1176 w & & & & $v$ ring $(5)$ \\
\hline & & & 1024 w & $1023 w$ & citrates \\
\hline & $997 \mathrm{vw}$ & 1001 vw & & & $v_{\text {as }} \mathrm{C}-\mathrm{O}$ (ribose) \\
\hline & $977 \mathrm{~m}$ & & & & $v_{\mathrm{s}} \mathrm{PO}_{3}{ }^{2-}$ \\
\hline & & & $952 w$ & $951 \mathrm{w}$ & citrates \\
\hline & $869 \mathrm{~m}$ & $880 w$ & $879 w$ & & $\delta$ ring $(6)$ \\
\hline & & $815 w$ & $812 \mathrm{~m}$ & $801 \mathrm{~m}$ & $\delta$ ring (6) \\
\hline & $669 \mathrm{~m}$ & $668 \mathrm{~s}$ & $669 \mathrm{~s}$ & $669 \mathrm{~m}$ & ring breathing \\
\hline & $584 w$ & $609 w$ & $606 w$ & & $\delta$ ring (6) \\
\hline & $501 w$ & $519 \mathrm{~m}$ & $515 \mathrm{~m}$ & $516 \mathrm{~m}$ & $\delta$ ring (6) \\
\hline & & 234 vs & & & $v \mathrm{Ag}-\mathrm{Cl}$ \\
\hline & & & 219 vs & 219 vs & $v \mathrm{Ag}-\mathrm{N} ; v \mathrm{Ag}-\mathrm{O}$ \\
\hline
\end{tabular}


Table 1. (continued)

\begin{tabular}{|c|c|c|c|c|c|}
\hline \multirow{3}{*}{$\begin{array}{c}\text { RNA } \\
\text { mononucleotide }\end{array}$} & \multirow{3}{*}{ Raman } & \multicolumn{3}{|c|}{ Wavenumber $/ \mathrm{cm}^{-1}$} & \multirow{3}{*}{ Assignment } \\
\hline & & & SERS & & \\
\hline & & Ag_Clnsp & Ag_cit nsp & Ag_cit nst & \\
\hline & 1606 vw & & 1633 w & $1628 \mathrm{w}$ & $v C=0$ \\
\hline & $1530 \mathrm{vw}$ & & 1492 w & & $v$ ring \\
\hline & & & 1393 w & $1391 \mathrm{w}$ & citrates \\
\hline & 1294 w & 1304 w & $1295 \mathrm{~m}$ & 1298 w & $v$ ring (N3-C4) \\
\hline & $1243 \mathrm{~m}$ & 1211 vw & $1219 w$ & $1219 w$ & $v$ ring $(\mathrm{N} 1-\mathrm{C} 2-\mathrm{N} 3)$ \\
\hline & & & $1025 \mathrm{~m}$ & 1028 w & citrates \\
\hline \multirow[t]{13}{*}{$\mathrm{CMP}$} & $978 m$ & & & & $v_{5} \mathrm{PO}_{3}{ }^{2-}$ \\
\hline & & & $949 w$ & $950 w$ & citrates \\
\hline & $871 \mathrm{vw}$ & & 877 vw & & $v$ ring \\
\hline & $782 \mathrm{~s}$ & $790 \mathrm{~m}$ & $787 \mathrm{~s}$ & $787 \mathrm{~m}$ & ring breathing \\
\hline & $597 w$ & $583 w$ & $600 w$ & & $\delta$ ring $(C 2-N 3-C 4)$ \\
\hline & & 237 vs & & & $v \mathrm{Ag}-\mathrm{Cl}$ \\
\hline & & & 221 vs & 219 vs & $v \mathrm{Ag}-\mathrm{N} ; v \mathrm{Ag}-\mathrm{O}$ \\
\hline & 1669 vw & & & & $v \mathrm{C}=0 ; v \mathrm{C} 5=\mathrm{C} 6$ \\
\hline & 1624 vw & & 1624 vw & & $v \mathrm{C}=\mathrm{O}$ \\
\hline & 1394 w & & & & $\delta_{\text {ip }} \mathrm{N} 3-\mathrm{H}$ \\
\hline & & & 1393 w & $1390 w$ & citrates \\
\hline & $1229 \mathrm{~s}$ & 1236 vw & & 1215 w & $v$ ring $(\mathrm{N} 1-\mathrm{C} 2-\mathrm{N} 3)$ \\
\hline & & & $1029 w$ & $1027 w$ & citrates \\
\hline \multirow[t]{7}{*}{ UMP } & $978 \mathrm{~m}$ & & & & $v_{\mathrm{s}} \mathrm{PO}_{3}{ }^{2-}$ \\
\hline & & & $950 w$ & $949 w$ & citrates \\
\hline & $783 \mathrm{~s}$ & $797 w$ & $792 \mathrm{~m}$ & $793 \mathrm{~m}$ & ring breathing \\
\hline & $645 \mathrm{vw}$ & & & & $\delta$ ring \\
\hline & $562 w$ & $577 w$ & $562 \mathrm{vw}$ & & $\delta$ ring $(\mathrm{C} 2-\mathrm{N} 3-\mathrm{C} 4)$ \\
\hline & & 237 vs & & & $v \mathrm{Ag}-\mathrm{Cl}$ \\
\hline & & & 219 vs & 223 vs & $v A g-N ; v A g-O$ \\
\hline
\end{tabular}

Abbreviations: vs, very strong; s, strong; $m$, medium; w, weak; vw, very weak; sh, shoulder; $v$, stretching; $\delta$, deformation; s, symmetrical; as, antisymmetrical; ip, in plane.

Number in brackets (5) and (6) indicates five-membered and six-membered ring, respectively.

coated silver nanospheres, while not obtained at all for any of the mononucleotides on the silver nanostars. Studies on SERS measurement of the anionic species on the citrate reduced silver nanoparticles implied that a competitive binding between the stabilizing anions and the anionic analytes with the silver surface take place, when the anion with the higher affinity for silver replaces the other one. ${ }^{[15]}$ It was also found that chlorides bind to the silver surface more strongly than citrates, ${ }^{[15]}$ indicating that the latter should be displaced by the analyte molecule more easily than the former. Given the poor SERS spectrum of AMP in the citrate coated colloid and the very weak SERS spectra of the RNA mononucleotides in the chloride coated colloid, the studied biomolecules had obviously a lower affinity for silver than both of the stabilizing surface anions. Another factor to consider was the surface coverage by the stabilizing anionic species dependent on their concentration and molecular structure. According to the nanoparticles preparation procedure, the chloride concentration was $2.4 \times 10^{-3}$ $\mathrm{mol} / \mathrm{L}$ in the colloidal suspension, which was higher than those of the biomolecules. On the other hand, the concentration of citrates was not known due to their participation in the reduction process. It was very likely that the bulky citrate ions prevented effective adsorption of the mono- 


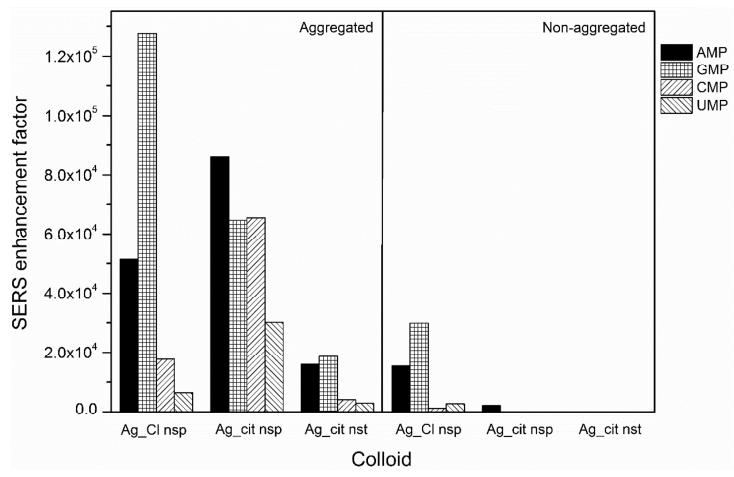

Figure 5. Comparison of the SERS enhancement factors for the RNA mononucleotides $\left(1 \times 10^{-5} \mathrm{~mol} / \mathrm{L}\right)$ on the aggregated and non-aggreagted silver colloids: Ag_Cl nsp, Ag_cit nsp and Ag_cit nst.

nucleotides onto the enhancing metal surface, hindering their approach to the nanoparticles, while there was a larger surface available for the interaction with the mononucleotides on the chloride stabilized nanospheres. ${ }^{[18]}$

The enhancement factors calculated for the RNA mononucleotides in the silver colloids $\left(1 \times 10^{-5} \mathrm{~mol} / \mathrm{L}\right)$ with and without the aggregating agent are compared in Figure 5. The $E F$ values of the order of $10^{4}$ were calculated for the chloride coated silver colloid, in absence of the aggregating agent. However, the scattered radiation was 2-10 times enhanced upon $\mathrm{Ag} \_\mathrm{Cl}$ nsp aggregation, showing a very similar trend with respect to the mononucleotides in the non-aggregated colloid. Interestingly, the SERS intensity of AMP increased as much as 40 times on the aggregated Ag_cit nsp, if compared to the colloid not containing the aggregating agent. Moreover, the Raman scattering of the other biomolecules on the aggregated citrated covered nanospheres was also obtained, which was for the pyrimidine mononucleotides, CMP and UMP, even more enhanced than on the aggregated chloride coated silver nanospheres. Consistent with the enhancement in the freshly prepared colloids, the weakest Raman scattering was observed from the aggregated silver nanostars. In general, the RNA mononucleotides intensively scattered radiation in all the sodium sulfate treated colloids, as a result of the nanoparticles aggregation. The addition of the aggregating agent surely affected the morphology of the nanoparticles as well as the chemical properties of the metallic surfaces, all contributing to the increased SERS activity. Owing to the formation of the larger aggregates, the surface plasmon resonance shifted towards longer wavelengths, being easily excited with the used laser radiation $(785 \mathrm{~nm}$ ). While inducing the aggregation of the silver nanoparticles, sodium cations most likely reduced the negative surface charge, leading to weaker repulsive forces between the silver nanoparticles and the

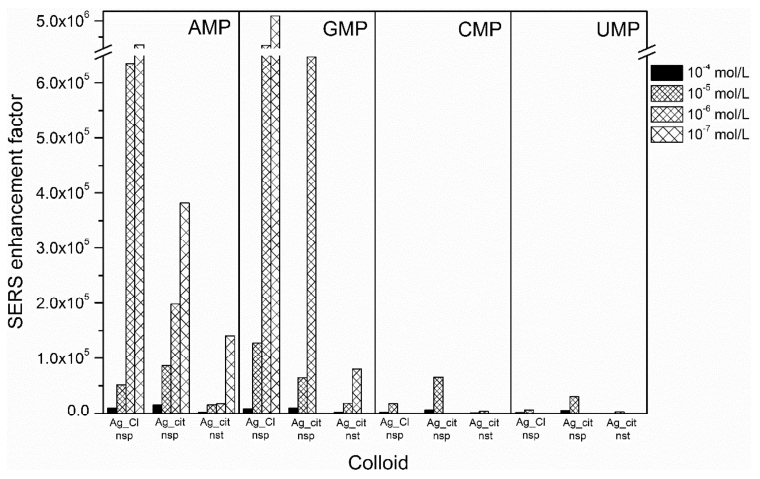

Figure 6. Comparison of the SERS enhancement factors for the RNA mononucleotides at various concentrations $\left(1 \times 10^{-4}\right.$, $1 \times 10^{-5}, 1 \times 10^{-6}$ and $1 \times 10^{-7} \mathrm{~mol} / \mathrm{L}$ ) on the aggregated silver colloids: Ag_Cl nsp, Ag_cit nsp and Ag_cit nst.

mononucleotides. Sulfate ions did not interfere with the measured biomolecules due to the low affinity of sulfates for the silver surface. ${ }^{[15]}$

Comparing the citrate coated nanoparticles of different shape, nanospheres and nanostars, a stronger enhancement had been expected from the star-shaped nanoparticles. A huge intensification of the electromagnetic field on the nanostars was suggested, particularly in the spaces between the star arms. ${ }^{6}$ Besides, the large extinction observed in the UV/Vis/NIR spectrum, implying the plasmon resonance in the NIR region, coincided with the wavelength of the laser excitation at $785 \mathrm{~nm}$. Nevertheless, the maximal $E F$, calculated for instance for AMP $\left(1 \times 10^{-6} \mathrm{~mol} / \mathrm{L}\right)$ on Ag_cit nanostars, was $3.4 \times 10^{4}$, which was almost one order of magnitude lower than the respective $E F$ value in $\mathrm{Ag}$ _cit nanospheres, reaching $2.3 \times$ $10^{5}$ (Figure 6).

Considering all the studied mononucleotides, the stronger enhancement was observed for AMP and GMP, than for CMP and UMP, regardless of the silver colloid (Figure 6). This could be attributed to the larger Raman cross-section of the condensed five- and six-membered rings of the purine mononucleotides, when compared to the single ring system of the pyrimidine mononucleotides, as well as to a greater number of the functionalities in the molecular structure of the purine bases capable of binding with the silver surface. Additionally, it was interesting to note that the Raman scattering of GMP was the most enhanced from $\mathrm{Ag} \_\mathrm{Cl}$ nsp at all the measured GMP concentrations, reaching up the $E F$ value of $5.8 \times 10^{6}$ $\left(1 \times 10^{-7} \mathrm{~mol} / \mathrm{L}\right)$, while it was concentration dependent for $\mathrm{AMP}$, being more intense from $\mathrm{Ag}$ cit $\mathrm{nsp}$ at higher AMP concentrations $\left(1 \times 10^{-5}\right.$ and $\left.1 \times 10^{-4} \mathrm{~mol} / \mathrm{L}\right)$, but from $\mathrm{Ag} \_\mathrm{Cl} n s p$ at lower AMP concentrations $\left(1 \times 10^{-7}\right.$ and $\left.1 \times 10^{-6} \mathrm{~mol} / \mathrm{L}\right)$. A difference in the enhancement factors, dependent on the molecular structure of the 
mononucleotide and the properties of the silver nanoparticles, could point to the different adsorption mechanisms of the molecules onto the enhancing metal surface. The adsorption of the RNA mononucleotides onto the citrate coated silver nanoparticles was studied in our previous work. ${ }^{16}$ The SERS spectra, obtained using NIR excitation, implied orientation of the molecules on the silver nanoparticles with the nucleic bases faced towards the metal surface, as sterically and electrostatically most convenient. Moreover, for the purine mononucleotides, AMP and GMP, adsorption through the larger aromatic ring was suggested, which was concentration dependent in case of AMP. Differing in applied excitation radiation, $1064 \mathrm{~nm}$ in our previous study and $785 \mathrm{~nm}$ in our current study, the obtained spectra did not fully coincide, as expected. Nevertheless, the position and relative intensity of the strongest SERS bands indicated the proposed adsorption mechanisms. Therefore, the binding with the surface of Ag_cit nst was mostly driven by interactions through the adenine N1 atom and $\mathrm{NH}_{2}$ group as well as via the guanine $\mathrm{N} 1 \mathrm{H}$ and $\mathrm{C}=\mathrm{O}$ group. Looking closely at the SERS spectra of the purine mononucleotides, obtained from $\mathrm{Ag} \_\mathrm{Cl} n s p$ and $\mathrm{Ag}$ _cit nsp, significant spectral changes were not observed (Figure 4). The position and relative intensity of the main vibrational bands in the SERS spectra obtained from different silver nanoparticles resembled each other, indicating very similar orientation of the molecules on both enhancing surfaces. Hence the differently enhanced scattering could be associated with the distance of the molecules from the silver surface, whereby at concentrations of $1 \times 10^{-5}$ and $1 \times 10^{-4}$ $\mathrm{mol} / \mathrm{L}$ AMP was placed closer to the citrate coated silver nanospheres, and GMP to the chloride coated ones. The SERS spectra of the pyrimidine mononucleotides, CMP and UMP, were rather weak, though more enhanced from the silver nanospheres with the citrate ions on the surface $\left(E F \approx 5 \times 10^{4}\right)$ than from the silver nanospheres with the surface layer of the chloride ions $\left(E F \approx 1 \times 10^{4}\right)$.

By decreasing the RNA mononucleotides concentration, the enhancement factors increased (Figure 6). The dependence of the calculated EFs on the mononucleotide concentration was associated with the surface coverage and surface selection rules. Hence the intensity of the SERS spectra depended on the orientation of the molecules towards the metal surface, whereby vibrations with polarizability change normal to the metal surface resulted in the strongest enhancement of the scattered radiation, and those parallel with the metal surface did not contribute to the scattering. At high concentration $\left(1 \times 10^{-4} \mathrm{~mol} / \mathrm{L}\right)$ the mononucleotide molecules were densely packed on the metal, resulting in a weaker enhancement due to either a slightly inclined position of the molecules on the metal surface or an ability to reabsorb scattered radiation from the neighboring molecules. Decreasing concentration a molecular monolayer was formed, whereby the molecules attained an optimal perpendicular orientation on the silver nanoparticles, producing the more intense spectra. A further concentration decrease caused a diminution in intensity due to a smaller number of the molecules able to lie flat on the enhancing surface. While the SERS spectra of AMP and GMP were observed down to concentration of $1 \times 10^{-7}$ $\mathrm{mol} / \mathrm{L}$, the spectra of CMP and UMP at the concentrations lower than $1 \times 10^{-5} \mathrm{~mol} / \mathrm{L}$ were not obtained. The vibrational bands distinctive of the pyrimidine mononucleotides disappeared from the spectra measured in $\mathrm{Ag} \_\mathrm{Cl} n s p$, and only the citrate bands contributed to the spectra acquired in the citrate covered colloids, Ag_cit nsp and Ag_cit nst. If compared to the Raman spectra of the aggregated citrate colloids (Figure 4), note that the weak citrates bands were also observed in the SERS spectra of the mononucleotides at the higher measured concentrations $\left(1 \times 10^{-5}\right.$ and $1 \times 10^{-4}$ $\mathrm{mol} / \mathrm{L})$, pointing to coexistence of the surface citrates and the biomolecules on the silver surface. Similarly, in the spectra measured on $\mathrm{Ag} \_\mathrm{Cl} \mathrm{nsp}$, a band around $237 \mathrm{~cm}^{-1}$, attributed to $\mathrm{Ag}-\mathrm{Cl}$ stretching vibration, ${ }^{19}$ indicated a simultaneous presence of the chloride ions on the silver nanoparticles, which were not displaced by the mononucleotide molecules.

To study the influence of the selected band for the $E F$ calculation, the enhancement factors were evaluated $\mathrm{u}$ sing two bands in the spectra of AMP and GMP. Beside the Raman bands at 728 and $669 \mathrm{~cm}^{-1}$, assigned to the sixmembered ring breathing of the nucleic bases, the EFs were calculated using the intensity of the bands at 1338 and 1322 $\mathrm{cm}^{-1}$, attributed to the stretching of the smaller, five-membered ring. In case of both, AMP and GMP, it was interesting to note that the slightly higher $E F$ values were observed when calculated with the band originating from the part of the molecule, presumably not involved directly in interactions with the silver surface (a column plot given only for AMP in Figure 7). However, a difference between the enhancement factors was not significant, not exceeding the factor of 2. Hence the EFs evaluated using intensity of the ring stretching bands were 1.6 and 1.8 times higher for AMP and GMP, respectively, on $\mathrm{Ag} \_\mathrm{Cl}$ nsp, and 1.1 to 1.4 times on Ag_cit nsp. Due to the increase of the spectral background the intensity of the bands around $1330 \mathrm{~cm}^{-1}$ could not be evaluated in the spectra measured at the lowest concentration of $1 \times 10^{-7} \mathrm{~mol} / \mathrm{L}$.

\section{CONCLUSION}

The enhancement efficiency of the silver nanoparticles, varying in the anionic surface layer and nanoparticle morphology, was studied for the SERS measurements of the negatively charged molecules. The analytical enhancement factors were calculated for four RNA 


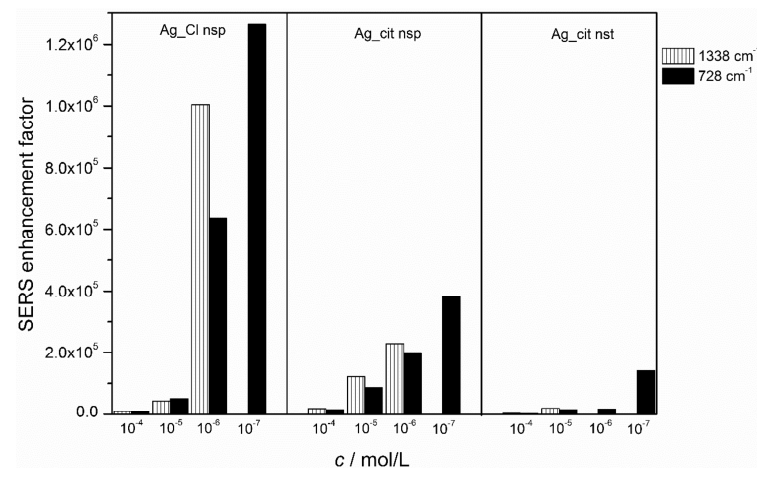

Figure 7. Comparison of the SERS enhancement factors for AMP at various concentrations $\left(1 \times 10^{-4}, 1 \times 10^{-5}, 1 \times 10^{-6}\right.$ and $1 \times 10^{-7} \mathrm{~mol} / \mathrm{L}$ ) on the aggregated silver colloids: $\mathrm{Ag} \_\mathrm{Cl} \mathrm{nsp}$, $\mathrm{Ag}$ _cit nsp and Ag_cit nst, calculated using intensity of the bands at 728 and $1338 \mathrm{~cm}^{-1}$.

mononucleotides, pointing to suitability of the studied silver colloids as the SERS active substrates. While a weak scattering was mostly observed from the non-aggregated silver nanoparticles, an addition of the inorganic salt into the silver colloids significantly increased their SERS activity. Hence in a series of the tested silver nanoparticles, the Raman scattering of the mononucleotides was strongly enhanced from the chloride coated nanospheres, moderately from the citrate covered nanospheres, and poorly from the citrate stabilized nanostars. Although characterized by plasmon resonances ranging from the blue to the near infrared region, the silver nanostars were the least effective metal substrates for the studied RNA mononucleotides. Apart from the silver nanoparticles properties, the enhancement factors depended on the mononucleotide concentration and molecular structure, which affected the adsorption, coverage and orientation of the molecules on the enhancing metallic surface. Nevertheless, the enhancement factors $10^{5}$ to $10^{6}$, calculated for the RNA mononucleotides on the aggregated silver nanospheres, were comparable to those reported for the cationic species on silver nanoparticles, implying a successful application of the spherically shaped nanoparticles in the SERS measurements of the negatively charged biomacromolecules.

\section{REFERENCES}

[1] R. Aroca, Surface-Enhanced Vibrational Spectroscopy, 2006, John Wiley \& Sons, Chichester.

[2] S. Schlücker, Surface-Enhanced Raman Spectroscopy: Analytical, Biophysical and Life Science Applications, 2011, Wiley-VCH, Weinheim.

[3] B. Sharma, R. R. Frontiera, A.-I. Henry, E. Ringe, R. P. Van Duyne, Mater. Today 2012, 15, 16.

[4] R. F. Aroca, R. A. Alvarez-Puebla, N. Pieczonka, S. Sanchez-Cortez, J. V. Garcia-Ramos, Adv. Colloid. Interfac. 2005, 116, 145.

[5] K. L. Kelly, E. Coronado, L. L. Zhao, G. C. Schatz, J. Phys. Chem. B 2003, 107, 668.

[6] A. Garcia-Leis, J. V. Garcia-Ramos, S. SanchezCortez, J. Phys. Chem. C 2013, 117, 7791.

[7] H. Bengter, C. Tengroth, S. P. Jacobsson, J. Raman Spectrosc. 2005, 36, 1015.

[8] L. Mikac, M. Ivanda, M. Gotić, T. Mihelj, L. Horvat, J. Nanopart. Res. 2014, 16, 2748.

[9] P. C. Lee, D. Meisel, J. Phys. Chem. 1982, 86, 3391.

[10] C. H. Munro, W. E. Smith, M. Garner, J. Clarkson, P. C. White, Langmuir 1995, 11, 3712.

[11] N. Leoplod, B. Lendl, J. Phys. Chem. B 2003, 107, 5723.

[12] E. Papadopoulou, S. E. J. Bell, Angew. Chem. Int. Ed. 2011, 50, 9058.

[13] E. Papadopoulou, S. E. J. Bell, Chem. Eur. J. 2012, 18, 5394.

[14] E. C. Le Ru, E. Blackie, M. Meyer, P. G. Etchegoin, J. Phys. Chem. C 2007, 111, 13794.

[15] S. E. J. Bell, N. M. S. Sirimuthu, J. Phys. Chem. A 2005, 109, 7405.

[16] S. Miljanić, A. Dijanošić, I. Matić, Spectrochim. Acta A 2015, 137, 1357.

[17] K. Nakamoto, M. Tsuboi, G. D. Strahan, Drug-DNA Interactions: Structures and Spectra, 2008, John Wiley \& Sons, New Jersey.

[18] M. V. Canamares, J. V. Garcia-Ramos, C. SanchezCortes, M. Castillejo, M. Oujja, J. Colloid Interf. Sci. 2008, 326, 103.

[19] E. J. Liang, C. Engert, W. Kiefer, Vib. Spectrosc. 1995, 8, 435. 